\title{
The relationship between trough concentration of vancomycin and effect on methicillin-resistant Staphylococcus aureus in critically ill patients
}

\author{
J Y Cheong, M Makmor-Bakry, C L Lau, R Abdul Rahman
}

Objectives. The incidence of methicillin-resistant Staphylococcus aureus (MRSA) infections in intensive care units in Malaysia is significant. Invasive MRSA infections are commonly treated with vancomycin. In clinical practice, the serum vancomycin trough concentration is used as a surrogate marker of vancomycin efficacy. A low concentration of vancomycin may result in less effective therapy and increase the risk of bacterial resistance. We evaluated the relationship between the resolution of MRSA infections and trough concentrations of vancomycin.

Methods. A total of 76 patients admitted between January 2005 and February 2011 were included in the study. Serum vancomycin trough concentration data were collected from the microbiology records. The clinical response was evaluated on the basis of clinical notes and culture test results.

Results. A total of 262 appropriate trough concentration data was included, with a median of 3 trough concentrations per patient.
Fifty-four patients responded to vancomycin therapy. The initial trough concentration did not differ between responders and nonresponders $(p=0.135)$, but the corrected trough concentration was higher among responders than among non-responders ( $11.64 \pm 1.50$ $\mathrm{mg} / \mathrm{l}$ and $9.25 \pm 1.59 \mathrm{mg} / \mathrm{l}$, respectively; $p=0.036$ ). The average total daily dose of vancomycin was significantly higher among the responders $(p=0.008)$.

Conclusion. In this critically ill population, a vancomycin dose of $15 \mathrm{mg} / \mathrm{kg} /$ day was found sufficient to produce optimal trough concentrations to eradicate the MRSA infection. This study demonstrated the significant relationship between response to treatment of MRSA infection and serum vancomycin trough concentrations.

S Afr Med J 2012;102(7):616-619.
Faculty of Pharmacy, Universiti Kebangsaan Malaysia, Kuala Lumpur, Malaysia J Y Cheong, BPharm (Hons), MClinPharm

M Makmor-Bakry, BPharm (Hons), MPharm (Clin), PhD

Department of Pharmacy, Universiti Kebangsaan Malaysia Medical Centre, Kuala Lumpur

C L Lau, BSc (Pharm), MClinPharm

Department of Anaesthesiology and Intensive Care, Universiti Kebangsaan Malaysia Medical Centre, Kuala Lumpur

R Abdul Rahman, MB BCh, BAO, MMed (Anaes)
Methicillin-resistant Staphylococcus aureus (MRSA) was first identified in Malaysia in early $1970 .{ }^{1}$ The MRSA infections rate was 10 per 1000 hospital admissions in a local teaching hospital between 2002 and $2007 .{ }^{2}$ More than $70 \%$ of S. aureus isolates in intensive care units (ICUs) in the USA are methicillin-resistant. ${ }^{3}$ Vancomycin (a glycopeptide antibiotic) has been used for nearly 50 years in treating MRSA infection. It remains first-line therapy against invasive MRSA infections. ${ }^{4}$ Vancomycin has a narrow therapeutic index and shows a time-dependent killing profile. ${ }^{5}$ Guidelines on recommended dosing of vancomycin have been published. ${ }^{4,6}$

The ratio of the area under the serum drug concentration-versustime curve (AUC) and the minimum inhibitory concentration (MIC) are among the most useful pharmacokinetic/pharmacodynamic 
parameters for predicting vancomycin efficacy. However, measurement of multiple serum concentrations for AUC calculation is not practical in the clinical setting; therefore, the serum trough concentration is used as a surrogate marker of AUC and represents the most practical method for monitoring the potential efficacy of vancomycin. Maximum killing occurs at sustained concentrations approximately 4 - 5 times above the MIC. ${ }^{5}$ In Malaysia, MRSA strains with higher MIC at $1 \mathrm{mg} / \mathrm{l}, 1.5 \mathrm{mg} / \mathrm{l}$ and $2 \mathrm{mg} / \mathrm{l}$ were found at the rate of $41 \%, 51 \%$ and $30 \%$, respectively, in 6 major hospitals. ${ }^{7}$ Low serum concentrations of vancomycin may result in less effective therapy and increased risk of bacterial resistance. ${ }^{8}$ Vancomycin resistance may be less likely to develop when serum trough concentrations are maintained above $10 \mathrm{mg} / \mathrm{l}^{9}$

Serum vancomycin trough concentrations of $15-20 \mathrm{mg} / \mathrm{l}$ have been recommended for severe MRSA infections such as bacteraemia, endocarditis, osteomyelitis, meningitis and hospital-acquired pneumonia. ${ }^{6}$ However, a trough concentration $>15 \mathrm{mg} / \mathrm{l}$ has not been clearly associated with favourable outcomes. ${ }^{10}$ An initial response rate of $74 \%$ was achieved when target trough concentrations of at least 4 times the MIC were attained. ${ }^{11}$ Vancomycin trough concentrations $>15 \mathrm{mg} / \mathrm{l}$ were also associated with a higher risk of nephrotoxicity. ${ }^{11}$

Little information is available regarding the clinical response of MRSA infections to serum vancomycin concentrations in critically ill patients. We evaluated the relationship between the resolution of MRSA infections and the trough concentrations of vancomycin and documented the dose regimen of vancomycin received by patients in the ICU.

\section{Methods}

This retrospective study of critically ill adult patients with MRSA infections was conducted in Universiti Kebangsaan Malaysia Medical Centre (UKMMC) by using the microbiology records from the Infectious Control Unit to identify all appropriate patients admitted between January 2005 and February 2011. This study was approved by the Research Ethics Committee of Universiti Kebangsaan Malaysia (UKM 1.5.3.5/244/NF-001-2011).

Inclusion criteria were: patients age $\geq 18$ years; who had microbiologically isolated MRSA; who were treated with vancomycin for $\geq 72$ hours; who had serum vancomycin trough concentrations monitored during therapy; whose baseline white blood cell (WBC) and neutrophil counts with $\geq 2$ serial readings were monitored during therapy; and whose baseline body temperature with serial body temperature readings during therapy were recorded. Patients concurrently treated with other antibiotics for MRSA infections (including linezolid, rifampicin, fusidic acid and teicoplanin) were excluded.

The medical and laboratory records of eligible patients were reviewed. Using a structured data collection form, demographic, laboratory, and clinical data were recorded, including: age, sex, body weight, diagnosis on admission to ICU, acute physiology and chronic health evaluation II (APACHE II) score on ICU admission, date of ICU admission, date of discharge from ICU and hospital, co-morbid conditions, WBC/neutrophil count, culture and sensitivity, daily body temperature, type of ventilation support, use of inotropic agents, vancomycin dosing regimen (dose and frequency), serum vancomycin trough concentrations achieved, and concurrent nonMRSA-sensitive antibiotics. The average value was used if there were more than one result per day for any laboratory investigation.

Information pertinent to vancomycin therapy was also collected, including dose, frequency, timing of doses, timing of missed doses, serum vancomycin concentrations, and timing of assay of the concentrations. An appropriate serum vancomycin trough concentration was defined as the concentration before the next scheduled vancomycin dose at steady-state level. All serum vancomycin concentrations were measured by fluorescence polarisation immunoassay (COBAS Integra 800 System, Roche, USA).

The dosage regimen of the patients was classified as 'stat dose basis' or 'multiple-dose basis'. Stat dose basis referred to a convenient method of dosing for patients with marked renal impairment, who are given an initial dose of at least $15 \mathrm{mg} / \mathrm{kg}$, followed by a maintenance dose of $250-1000 \mathrm{mg}$ once every few days based on the measurement of serum trough concentrations. Multiple-dose basis is the traditional method of giving a dose at a fixed frequency, e.g. 8-hourly, 12-hourly and 24-hourly. 12,13

A primary outcome measure was clinical response at the end of vancomycin therapy. Clinical responses were evaluated daily after 72 hours of vancomycin administration. Based on the clinical outcome, subjects were divided into 'response' and 'non-response' groups. 'Response' was defined as improvement of infection-related parameters (such as decreasing WBC/neutrophil count, improvement of local signs of infections, decreasing body temperature, de-escalation of the use of invasive ventilation support to non-invasive ventilation support, and discontinuation of inotropic agents, with or without negative culture of MRSA at the end of vancomycin treatment). 'Non-response' was defined as no improvement, or worsening of signs and symptoms of infection, with or without persistent positive culture, or a switch from vancomycin to an alternative agent based on the clinical judgement of clinicians and with or without persistent positive culture.

Initial serum vancomycin trough concentration referred to the first steady-state trough concentration. For each patient, a corrected average serum vancomycin trough concentration was calculated as the trough concentration achieved using the sum of each measured trough concentration multiplied by the number of days at that level, then divided by the total number of treatment days. ${ }^{11}$ Clinical outcomes for all patients were analysed using the initial vancomycin trough concentration achieved and the corrected serum trough concentration.

\section{Results}

Of 217 patients with MRSA infection and treated with vancomycin, only 112 had serum trough concentrations available. Of these patients, 76 fulfilled the inclusion and exclusion criteria. Table 1 shows overall patient demographic data. Male patients comprised $75 \%$. On admission, the APACHE II score was $18.76 \pm 8.18$ $($ mean \pm SD). Most isolates were obtained from the respiratory tract $(46(60.5 \%))$. There were $17(22.4 \%)$ patients with multiple sites of MRSA infection, and $93.4 \%$ patients had other concurrent infections. All patients with concurrent infections were treated with other antibiotics based on culture and sensitivity tests and appropriate dose regimen. A total of 262 appropriate trough concentration data were included in the study, with a median of 3 trough concentrations per patient.

Responders to vancomycin therapy $(N=54)$ were categorised into 2 groups: those who responded clinically with negative culture at the end of therapy $(n=44)$, and those who responded clinically but without culture result at the end of therapy $(n=10)$. Non-responders to therapy $(n=22)$ were classified as: clinically not improved with persistent positive culture $(n=8)$, clinically not improved without culture result $(n=6)$, changed to alternative agent with persistent positive culture $(n=7)$, or changed to alternative agent without culture result $(n=1)$.

Table 2 shows patient demographics and serum vancomycin trough concentrations for responders and non-responders. All aspects of demographic data were similar in the 2 groups. Initial trough concentrations did not differ between responders and non- 
Table 1. Demographic description of the study subjects

\begin{tabular}{|c|c|}
\hline Variables & $\begin{array}{l}\text { Value } \\
(N=76)\end{array}$ \\
\hline Age, years $($ mean $\pm S D)$ & $55.5 \pm 16.6$ \\
\hline Weight, kg (median (IQR)) & $68.4(56.9-80.0)$ \\
\hline \multicolumn{2}{|l|}{ Gender } \\
\hline Male & $57(75 \%)$ \\
\hline Female & $19(25 \%)$ \\
\hline \multicolumn{2}{|l|}{ ICU admission diagnosis } \\
\hline Stabilisation pre/post operation & $25(32.9 \%)$ \\
\hline Respiratory failure & $44(57.9 \%)$ \\
\hline Reduced Glasgow coma scale & $7(9.2 \%)$ \\
\hline \multicolumn{2}{|l|}{ Co-morbid conditions } \\
\hline Hypertension & $42(55.3 \%)$ \\
\hline Cerebrovascular accident & $6(7.9 \%)$ \\
\hline Pulmonary disease & $19(25 \%)$ \\
\hline Diabetes mellitus & $27(35.5 \%)$ \\
\hline Cardiovascular disease & $17(22.4 \%)$ \\
\hline Renal disease & $29(38.2 \%)$ \\
\hline Liver disease & $15(19.7 \%)$ \\
\hline Trauma & $19(25 \%)$ \\
\hline Surgery & $45(59.2 \%)$ \\
\hline Malignancy & $11(14.5 \%)$ \\
\hline Gastro-intestinal disease & $7(9.2 \%)$ \\
\hline Premorbid obesity & $5(6.6 \%)$ \\
\hline APACHE II score (mean \pm SD) & $18.76 \pm 8.18$ \\
\hline \multicolumn{2}{|l|}{ Infection site } \\
\hline Respiratory & $46(60.5 \%)$ \\
\hline Blood & $5(6.6 \%)$ \\
\hline Wound & $8(10.5 \%)$ \\
\hline Multiple & $17(22.4 \%)$ \\
\hline \multicolumn{2}{|c|}{ Baseline serum creatinine, $\mathrm{mmol} / \mathrm{l}$ (median } \\
\hline Baseline $\mathrm{CrCl}, \mathrm{ml} / \mathrm{min}(\operatorname{mean} \pm \mathrm{SD})$ & $80.07 \pm 9.11$ \\
\hline \multicolumn{2}{|l|}{ Vancomycin dosing type } \\
\hline Multiple-dose basis & $58(76.3)$ \\
\hline Stat dose basis & $18(23.7)$ \\
\hline $\begin{array}{l}\text { Concentration readings per patient } \\
\text { (median (IQR)) }\end{array}$ & $3(2-4)$ \\
\hline \multicolumn{2}{|l|}{ Vancomycin trough concentrations } \\
\hline Initial, $\mathrm{mg} / \mathrm{l}(\mathrm{mean} \pm \mathrm{SD})$ & $9.69 \pm 1.68$ \\
\hline Corrected, mg/l (mean \pm SD) & $10.89 \pm 1.54$ \\
\hline
\end{tabular}

responders $(p=0.135)$ but the corrected trough concentrations were higher among responders $(p=0.036)$. There was no difference between the responders and non-responders distribution as regards patients on multiple-dose basis and stat dose basis. The average total daily dose was significantly higher in the responders $(p=0.008)$.

\section{Discussion}

The initial and corrected vancomycin trough concentrations were higher among responders than non-responders, but statistical significance was only observed in corrected trough concentrations.
This could be explained by the time-dependent bacterial killing characteristic of vancomycin. The corrected trough concentration was estimated by using all measured trough concentrations during the entire course of therapy instead of only one measurement of initial trough concentration. As such, the corrected trough concentration gives a better picture of the vancomycin trough concentration throughout the course of therapy. This finding implies that serum vancomycin trough concentrations should be monitored throughout the course of therapy in critically ill patients. Studies have found inter-individual variability in vancomycin disposition in critically ill patients owing to factors such as age, renal function and underlying illnesses. ${ }^{14,15}$ Concurrent use of haemodynamically active drugs such as inotropes and furosemide have been suggested as enhancing vancomycin clearance in this population. ${ }^{14}$ Therefore, close monitoring of trough concentrations is imperative in adjusting vancomycin dosage to optimise the treatment effect.

The corrected trough concentration of $11.64 \pm 1.50 \mathrm{mg} / \mathrm{l}$ among the responders compared with $9.25 \pm 1.59 \mathrm{mg} / \mathrm{l}$ among non-responders showed the importance of maintaining vancomycin trough concentration above $10 \mathrm{mg} / \mathrm{l}$ throughout the course of therapy for optimal efficacy. As the maximum killing effect of vancomycin depends on sustained concentrations 4 - 5 times above the MIC, it is postulated that there is a higher prevalence of MRSA strains with higher MIC in the present study location, and may be similar to that in other major hospitals in Malaysia. ${ }^{7}$ Similar findings were reported by Zimmermann et al. ${ }^{16}$ who found that patients were more likely to have WBC counts return to normal and become afebrile within 72 hours if trough concentrations of vancomycin were $10 \mathrm{mg} / \mathrm{l}$ or above. ${ }^{16}$ This finding also supports other reports that trough concentration of vancomycin should be maintained above $10 \mathrm{mg} / \mathrm{l}$ for treatment to be successful and to prevent the development of vancomycin resistance. ${ }^{9}$

The doses of vancomycin given to the critically ill patients were determined and adjusted according to the patient's renal function, creatinine clearance and the vancomycin trough concentration achieved with the earlier dose. Based on our study findings, a vancomycin dose of $15 \mathrm{mg} / \mathrm{kg} / \mathrm{day}$ would be sufficient to produce optimal trough concentrations to eradicate MRSA. This dose was estimated to produce the corrected vancomycin trough concentration of $11.64 \pm 1.50 \mathrm{mg} / \mathrm{l}$, which is between 10 and $15 \mathrm{mg} / \mathrm{l}$ as previously recommended. ${ }^{9}$ Others also failed to demonstrate the benefits of recently recommended higher serum vancomycin trough concentrations $>15 \mathrm{mg} / \mathrm{l}$ in achieving better clinical outcomes. ${ }^{10,11,17}$ The current findings may justify the vancomycin trough concentration between 10 and $15 \mathrm{mg} / \mathrm{l}$ as an effective and safe target for MRSA infection in critically ill patients. However, this observation has not been interpreted in the presence of MICs, and caution must be exercised before applying this finding to other study populations.

In conclusion, this study demonstrated the significant relationship between treatment response of MRSA infection and serum vancomycin trough concentrations. In our critically ill adult population, a vancomycin dose of $15 \mathrm{mg} / \mathrm{kg} /$ day would be sufficient to produce an optimal trough concentration of $10-15 \mathrm{mg} / \mathrm{l}$ that is effective against MRSA.

\section{References}

1. Lim VKE, Zulkifli HI. Methicillin resistant Staphylococcus aureus in a Malaysian neonatal unit. Singapore Med J 1987;28:176-179.

2. Al-Talib HI, Yean CY, Al-Jashamy K, Hasan H. Methicillin-resistant Staphylococcus aureus nosocomial infection trends in Hospital Universiti Sains Malaysia during 2002-2007. Ann Saudi Med 2010;30(5):358-363. 
RESEARCH

Table 2. Patient demographics and serum trough concentrations in responders and non-responders to vancomycin therapy

\begin{tabular}{|c|c|c|c|}
\hline Variables & $\begin{array}{l}\text { Responders } \\
(n=54)\end{array}$ & $\begin{array}{l}\text { Non-responders } \\
(n=22)\end{array}$ & $p$-value \\
\hline \multicolumn{4}{|l|}{ Patient demographics } \\
\hline Age, years $($ mean $\pm S D)$ & $53.56 \pm 16.08$ & $60.41 \pm 16.91$ & 0.101 \\
\hline Weight, kg (median (IQR)) & $69.9(59.9-82.0)$ & $64.4(55.1-75.8)$ & 0.149 \\
\hline \multicolumn{4}{|l|}{ Gender } \\
\hline Male & $43(79.6)$ & $14(63.6)$ & \\
\hline Female & $11(20.4)$ & $8(36.3)$ & 0.144 \\
\hline APACHE II score (mean \pm SD) & $18.89 \pm 8.50$ & $18.45 \pm 7.51$ & 0.835 \\
\hline \multicolumn{4}{|l|}{ Length of stay after initiation of vancomycin (d) } \\
\hline $\mathrm{ICU}($ mean $\pm \mathrm{SD})$ & $10.06 \pm 3.47^{\dagger}$ & $20.37 \pm 2.42 \ddagger$ & 0.198 \\
\hline Hospital $($ mean \pm SD) & $33.52 \pm 2.57^{\dagger}$ & $49.59 \pm 2.00 \ddagger$ & 0.344 \\
\hline \multicolumn{4}{|l|}{ Vancomycin trough concentration } \\
\hline Initial, $\mathrm{mg} / \mathrm{l}(\mathrm{mean} \pm \mathrm{SD})$ & $10.26 \pm 1.69$ & $8.42 \pm 1.64$ & 0.135 \\
\hline Corrected, mg/l (mean $\pm \mathrm{SD})$ & $11.64 \pm 1.50$ & $9.25 \pm 1.59$ & $0.036^{*}$ \\
\hline \multicolumn{4}{|l|}{ Vancomycin dosing information } \\
\hline \multicolumn{4}{|l|}{ Dosing basis } \\
\hline Multiple-dose & $43(79.6)$ & $15(68.2)$ & \\
\hline Stat dose & $11(20.4)$ & $7(31.8)$ & 0.287 \\
\hline Loading dose given & $9(20.9)^{\varsigma}$ & $1(6.7)^{9}$ & 0.388 \\
\hline Initial total daily dose, $\mathrm{mg} / \mathrm{kg} /$ day $($ mean $\pm \mathrm{SD})$ & $18.80 \pm 1.70$ & $14.10 \pm 1.75$ & $0.039^{*}$ \\
\hline Average total daily dose, $\mathrm{mg} / \mathrm{kg} /$ day $($ mean $\pm \mathrm{SD})$ & $15.17 \pm 1.99$ & $9.42 \pm 1.97$ & $0.008^{\star}$ \\
\hline Cumulative dose received, $\mathrm{g}($ mean $\pm \mathrm{SD})$ & $10.89 \pm 2.30$ & $4.71 \pm 1.77$ & $<0.001^{*}$ \\
\hline Duration of therapy, days (mean $\pm S D)$ & $10.10 \pm 1.68$ & $7.62 \pm 1.77$ & $0.041^{*}$ \\
\hline \multicolumn{4}{|l|}{ Data are given as number (percentage) unless otherwise indicated. } \\
\hline \multicolumn{4}{|l|}{ "Statistically significantly different. } \\
\hline \multicolumn{4}{|l|}{${ }_{n=29}$ (total responders who survived). } \\
\hline \multicolumn{4}{|l|}{${ }_{n=6}^{*}$ (total non-responders who survived). } \\
\hline$n=43$ (total responders who were on multiple-dose basis). & & & \\
\hline ' $n=15$ (total non-responders who were on multiple-dose basis). & & & \\
\hline
\end{tabular}

3. Klevens RM, Edwards JR, Tenover FC, McDonald LC, Horan T, Gaynes R. Changes in the epidemiology of methicillin-resistant Staphylococcus aureus in intensive care units in US hospitals, 1992-2003. Clin Infect Dis 2006:42(3):389-391.

4. Liu C, Bayer A, Cosgrove SE, et al. Clinical practice guidelines by the Infectious Diseases Society of America for the treatment of methicillin-resistant Staphylococcus aureus infections in adults and children. Clin Infect Dis 2011;52(3):e18-e55.

5. Mueller M, Pena A, Derendorf H. Issues in pharmacokinetics and pharmacodynamics of anti-infective agents: kill curves versus MIC. Antimicrob Agents Chemother 2004;48(2):369-377.

6. Rybak M, Lomaestro B, Rotschafer IC, et al. Therapeutic monitoring of vancomycin in adult patients: a consensus review of the American Society of Health-System Pharmacists, the Infectious Diseases Society of America, and the Society of Infectious Diseases Pharmacists. Am J Health Syst Pharm 2009;66(1):82-98. 7. Ahmad N, Nawi S, Rajasekaran, et al. Increased vancomycin minimum inhibitory concentration among Staphylococcus aureus isolates in Malaysia. J Med Microbiol 2010;59(12):1530-1552. 8. de Hoog M, Mouton JW, van den Anker JN. Vancomycin: pharmacokinetics and administration

9. Sakoulas G, Gold HS, Cohen RA, Venkataraman L, Moellering RC, Eliopoulos GM. Effects of prolonged vancomycin administration on methicillin-resistant Staphylococcus aureus (MRSA) in a patient with recurrent bacteraemia. J Antimicrob Chemother 2006;57(4):699-704.

10. Lodise TP, Graves I, Evans A, et al. Relationship between vancomycin MIC and failure among patients with methicillin-resistant Staphylococcus aureus bacteremia treated with vancomycin. Antimicrob Agents Chemother 2008;52(9):3315-3320.
11. Hidayat LK, Quist R, Shriner KA, Wong-Beringer A. High-dose vancomycin therapy for methicillinresistant Staphylococcus aureus infections - efficacy and toxicity. Arch Intern Med 2006;166(19):2138-2144 12. Eli Lilly \& Company. Vancocin package insert, 2003.

3. Hospira Incorporation. Sterile vancomycin hydrochloride USP package insert, 2008 .

14. Pea F, Porreca L, Baraldo M, Furlanut M. High vancomycin dosage regimen required by intensive care unit patients cotreated with drugs to improve hemodynamics following cardiac surgical procedures. Antimicrob Chemother 2000;45(3):329-335.

15. del Mar Fernández de Gatta Garcia M, Revilla N, Calvo MV, Domínguez-Gil A, Navarro AS Pharmacokinetic/pharmacodynamic analysis of vancomycin in ICU patients. Intensive Care Med 2007;33(2):279-285

16. Zimmermann AE, Katona BG, Plaisance KI. Association of vancomycin serum concentrations with outcomes in patients with gram-positive bacteraemia. Pharmacotherapy 1995;15(1):85-91.

7. Jeffres MN, Isakow W Doherty JA, et al. Predictors of mortality for methicillin-resistant Staphylococcus aureus health care-associated pneumonia: specific evaluation of vancomycin pharmacokinetic indices. Chest 2006;130(4):947-955.

Accepted 6 January 2012 